\title{
Transpupillary thermotherapy for atypical central serous chorioretinopathy
}

This article was published in the following Dove Press journal:

Clinical Ophthalmology

24 January 2012

Number of times this article has been viewed

\section{Ryosuke Kawamura ${ }^{1,2}$ \\ Hidenao Ideta' \\ Hideyuki Hori' \\ Kenya Yuki ${ }^{2}$ \\ Tsuyoshi Uno' \\ Tatsurou Tanabe' \\ Kazuo Tsubota ${ }^{2}$ \\ Tsutomu Kawasaki ${ }^{1}$}

'Ideta Eye Hospital, Kumamoto, Japan; ${ }^{2}$ Keio University, School of Medicine, Department of Ophthalmology, Tokyo, Japan
Correspondence: Ryosuke Kawamura 35, Shinanomachi, Shinjuku, Tokyo, Japan, I60-8582

Tel $+8 \mid 33353$ I 2 II ext 62402

Fax +8I 353633869

Email ryosuke@wc4.so-net.ne.jp
Background: Central serous chorioretinopathy (CSC) has been traditionally treated with laser photocoagulation. We thought that transpupillary thermotherapy (TTT) utilizing a lower temperature than that of conventional laser photocoagulation might minimize permanent retinal and choroidal damage. Studies suggest that undesirable effects on vision due to TTT are minimal even if it is applied to foveal and/or parafoveal lesions when TTT requires a larger irradiation spot. The aim of this study was to evaluate the efficacy of TTT in the management of atypical CSC.

Methods: We defined atypical CSC as bullous retinal detachment with diffuse or several leakages, severe leakage with fibrin formation under serous retinal detachment, or leakage within a pigment epithelium detachment. Eight consecutive patients with atypical CSC underwent visual acuity testing, ophthalmic examination, color photography, fluorescein angiography, and optical coherence tomography to evaluate the results of transpupillary thermotherapy. Retreatment of atypical CSC was based on ophthalmic examination, optical coherence tomography, and fluorescein angiography. TTT was performed on the leaking spots shown in fluorescein angiography, with a power of 50-250 mW, spot size of 500-1200 $\mu \mathrm{m}$, and exposure time of 13-60 seconds to minimize retinal damage.

Results: In five of eight affected eyes, serous detachments completely resolved within 1 month after the initial TTT. One eye had persistent subretinal fluid and required a second TTT treatment. Two eyes showed no resolution of CSC and were treated by conventional photocoagulation. Initial best-corrected visual acuity (BCVA) ranged from 20/600 to 20/20 (mean, 20/40; median, 20/30). Final BCVA ranged from 20/200 to 20/20 (mean, 20/25; median, 20/20). BCVA improved in all cases. Only two eyes with persistent subretinal fibrin and existing retinal pigment epithelial alternations in macular area showed limited improvement of BCVA despite the absence of subretinal exudation. The presence of retinal attachment was confirmed by optical coherence tomography in six eyes (75\%).

Conclusions: TTT seems to be effective for the treatment of atypical CSC in the short term. Additional studies are necessary to evaluate the long-term effectiveness and safety.

Keywords: transpupillary thermotherapy, central serous chorioretinopathy, optical coherence tomography, fluorescein angiography, serous detachment

\section{Introduction}

Transpupillary thermotherapy (TTT) was introduced in 1992 as a new treatment for choroidal melanoma. ${ }^{1}$ TTT delivers heats to the target tissues in the form of a laser beam using a lower power for a prolonged period to limit damage to surrounding tissues. This technique contrasts with the laser used in conventional photocoagulation therapy in that the conventional laser treatment uses a high power for a short period of time, causing 
tissue damage. Since TTT was first performed for choroidal melanoma, ${ }^{1}$ the application of TTT has been expanded to other malignant tumors including hemangioma, ${ }^{2,3}$ and choroidal neovascularization in age-related macular degeneration (AMD). ${ }^{4,5}$

Central serous chorioretinopathy (CSC) has been traditionally treated with laser photocoagulation. Although recent articles have reported that TTT can be also utilized for the treatment of CSC, ${ }^{6,7}$ we thought that TTT utilizing a lower temperature than that of conventional laser photocoagulation might minimize permanent retinal and choroidal damage. Conventional photocoagulation results in the formation of scars in the retinal pigment epithelium (RPE). It can be applied only when retinal detachment is minimal and fluorescein leaking points are few. Therefore it has limited use in the treatment of chronic and atypical or excessive CSC when retinal detachment is excessive and leaking points are multiple. However TTT can be applicable in those cases. Tranos has reported that after the TTT treatment of subfoveal neovascularization in AMD, vision is either maintained or improved in more than $70 \%$ of cases. ${ }^{8}$ This suggests that the undesirable effects on vision by TTT are minimal even if it is applied to foveal and/or parafoveal lesions when TTT requires a larger irradiation spot.

There is still no established therapy for atypical CSC. Although selected cases of acute CSC benefit from retinal laser photocoagulation, there is no standard treatment for atypical CSC. ${ }^{9,10}$ In this small pilot study, we evaluated the use of TTT in the management of atypical CSC.

This report is proof of the concept that TTT is at least effective in the short term in the treatment of serous detachment associated with atypical CSC.

\section{Subjects and methods}

The study followed the Tenets of the Declaration of Helsinki. This study was a retrospective, uncontrolled interventional case series of consecutive patients with atypical CSC treated by TTT at Ideta Eye Hospital, Kumamoto, Japan. Patients who visited the hospital and were diagnosed with atypical CSC from April 2004 through December 2006 were included in this study. This study was performed with approval from the Ethic Board Committee of Ideta Eye hospital.

Inclusion/exclusion criteria were as follows:

Inclusion criteria:

1. Patients selecting TTT as their first therapy.

2. CSC patients who had documented recurrences and hoped for early recovery.

3. Duration of CSC exceeding 3 months.
4. Presence of excessive retinal detachment.

5. Presence of multiple leaking points in fluorescein angiography (FA).

6. Presence of macular pigment epithelium detachment (PED) in association with CSC.

Exclusion criteria:

1. Patients selecting conventional photocoagulation as their first therapy.

2. Presence of a subfoveal leakage point in FA.

3. Possibility of choroidal neovascularization in FA and/or indocyanine green angiography (IA).

The diagnosis of CSC was based on clinical appearance, and the findings from FA, IA, and optical coherence tomography (OCT).

\section{Treatment protocol}

TTT was performed under topical anesthesia under mydriasis. A slit-lamp microscope was attached to a diode laser employing near infrared diode laser radiation at a wavelength of $810 \mathrm{~nm}$. The laser parameter was determined based on the protocol for choroidal neovascularization. The power setting was set $10 \%$ lower than that of the protocol for choroidal neovascularization, ${ }^{4}$ because CSC has no proliferative tissues and we observed scar formation in case 1, which was the first case treated by TTT. The spot sizes were determined by FA. The hyperfluorescent areas with leaking spots and ooze were included.

\section{Outcome measurement}

The treatment outcome was assessed by resolution of the serous retinal detachment on fundus examination through slitlamp, leakage on FA, OCT, and best-corrected visual acuity (BCVA). If the retinal detachment and the angiographic leakage persisted more than 2 months after the treatment, re-treatment was performed.

\section{Results}

The study included eight eyes of eight males. Their mean age was $51.9 \pm 12.1$ years (range 39-72 years). Duration from onset of visual impairment to TTT ranged from 3-12 months (mean; 7 months).

Initial BCVA ranged from 20/600 to 20/20 (mean; logMAR BCVA -0.60, median; 20/30). The mean follow-up period after TTT was 4.5 months (2-10 months) and BCVA at final follow-up ranged from 20/200 to 20/20 (mean; logMAR BCVA -0.21, median; 20/20).

Anatomical success with complete resolution of serous retinal detachment was obtained in five eyes 
(cases 1, 2, 3, 4, and 7) on fundus examination and OCT. One eye (case 5) showed only a decrease of serous retinal detachment with persistence of subretinal exudation lasting for 2 months. After the second TTT treatment the retina was reattached. Two eyes (cases 6 and 8) showed no resolution. After discussions with the patients, these two eyes were treated by conventional photocoagulation. Finally, anatomical success was assessed in all eyes.

BCVA improved in all patients and retinal attachment was achieved in six eyes (75\%) (cases 2, 3, 4, 6, 7 and 8). There were two cases that showed no visual improvement despite the absence of subretinal fluid. One case (case 1) showed persistent subretinal fibrin while the other case (case 5) had RPE alternation in macula as demonstrated by OCT.

None of the cases developed severe complications except for extrafoveal scar formation close to the area of irradiation in one eye (case 1). In two cases, the irradiation was discontinued before completing the planned 60-second exposure time. In one case (case 1), irradiation was terminated at 13 seconds because of grayish retinal discoloration and the other case (case 2) was stopped at 45 seconds because of pain.

\section{Case reports Case I (Figure I)}

A 39-year-old male complained of decreased visual acuity for 2 months. He had a history of CSC that spontaneously cured. Best corrected visual acuity was 20/300 in his left eye at his first visit. The fundus examination revealed subretinal exudation and subretinal fluid (2 disk-diameters in size) in the macula. TTT was performed at the leakage point in FA. TTT was performed with a spot size of $1200 \mu \mathrm{m}$ at a power of $250 \mathrm{~mW}$. Because retinal color changed during the irradiation, TTT was stopped at 13 seconds. One month after the TTT treatment, fundus examination and OCT revealed resolution of exudation and subretinal fluid. However, extrafoveal scar formation of the irradiated area developed. Four months after the treatment, his visual acuity was 20/200.

Because of this scar formation in case 1, who was the first case treated by TTT, we reduced the power to $90 \%$ to deliver a subthreshold retinal burn. We also checked the level of burn by performing test spots in the inferonasal quadrant.

\section{Case 2 (Figure 2)}

A 45-year-old male complained of a defect in his visual field for 1 month. Best corrected visual acuity was 20/20 in his left eye. Fundus examination showed an inferior bullous retinal detachment with shifting fluid and a subretinal exudation at

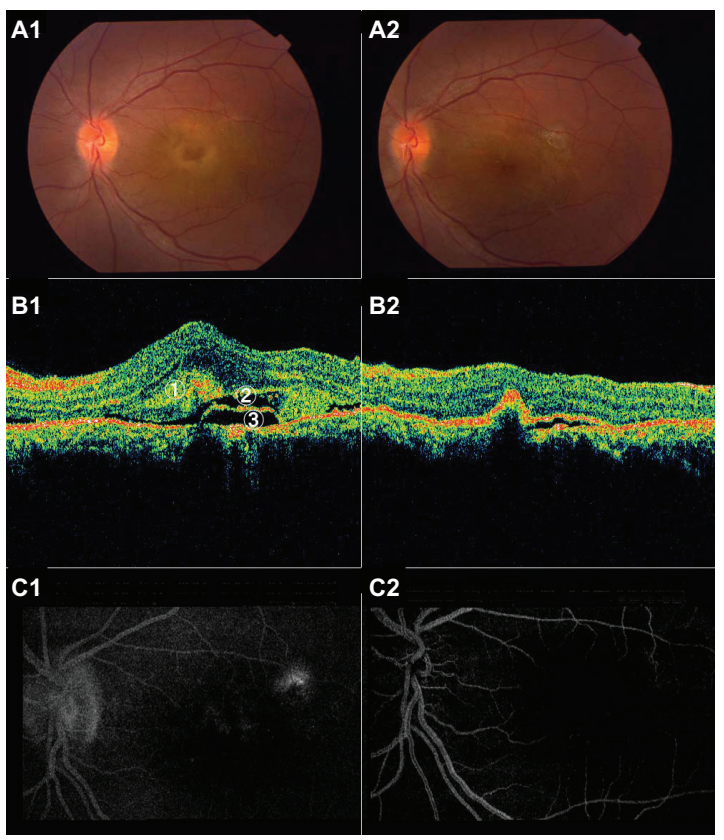

Figure I Case I: Color fundus photography shows serous detachment and yellowish exudates under the retina in the macular area (AI). OCT shows subretinal fibrin, subretinal fluid, and pigment epithelial detachment before treatment (BI). Fluorescein leaking point is temporal to the macular lesion $(\mathbf{C I})$. Post-treatment color photograph shows no serous detachment (A2). OCT shows no retinal detachment but minimal RPE detachment and extrafoveal scar formation at the irradiation area (B2). Fluorescein leakage had subsided (C2).

Abbreviations: OCT, optical coherence tomography; RPE, retinal pigment epithelium.

the inferotemporal area, where FA showed two leak points. TTT was performed with $800 \mu \mathrm{m}$ at $230 \mathrm{~mW}$ for 45 seconds for each spot. One month after the treatment, subretinal fluid diminished. Three months later, visual acuity was 20/20.

\section{Case 3 (Figure 3)}

A 45-year-old male complained of visual loss for 1 month. Best corrected visual acuity was 20/600. There were three retinal pigment epithelium detachments (PED) and serous retinal detachment superiorly. FA showed the leakage point in PED superior to the optic disk. TTT was performed at the leakage point in PED with a spot size of $500 \mu \mathrm{m}$ and a power of $50 \mathrm{~mW}$ for 60 seconds. The subretinal fluid disappeared within 2 weeks. Three months later, visual acuity was $20 / 100$.

\section{Discussion}

The current concept of underlying pathology in CSC is abnormal choroidal hyperpermeability. Typically, one or more discrete isolated leaks are detected at the level of the retinal pigment epithelium (RPE) in FA. Figure 1A also demonstrates multifocal choroidal hyperpermeability following focal hypofluorescent lesions. This suggests that the 


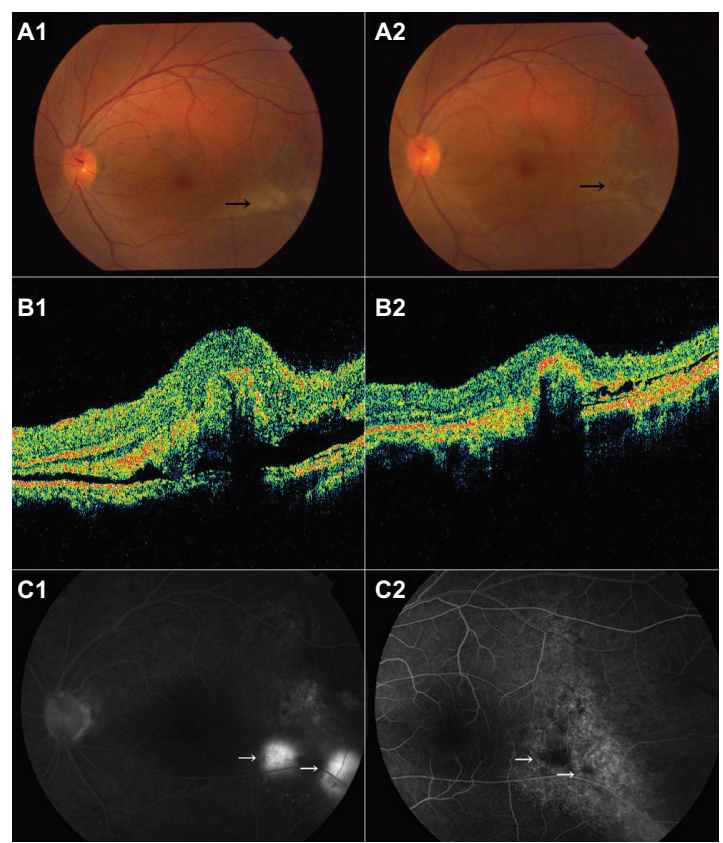

Figure 2 Case 2: Color fundus photography shows yellowish lesion inferotemporal to the fovea (black arrow) (AI). OCT shows subretinal fluid with fibrin and possible RPE elevation (BI). Fluorescein angiography shows two leakage points (white arrow) (CI). Post-treatment color photograph shows no yellowish exudate (black arrow) (A2). OCT shows decreased subretinal fluid and RPE detachment (B2). Fluorescein leakage had subsided (C2).

Abbreviations: OCT, optical coherence tomography; RPE, retinal pigment epithelium.

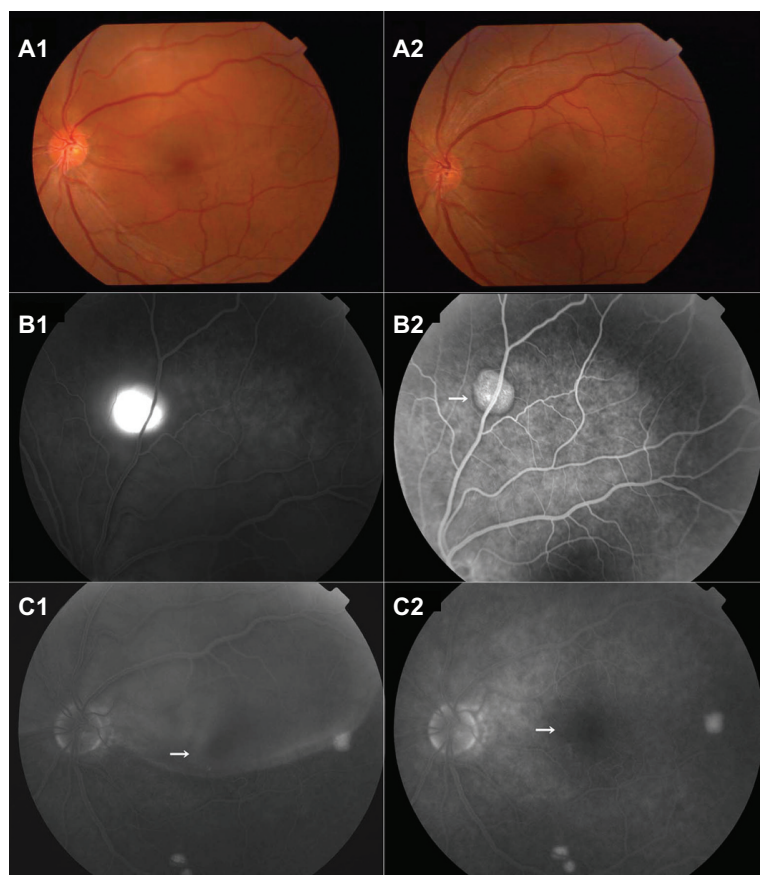

Figure 3 Case 3: Color photography shows bullous retinal detachment located superior to the disk and fovea (AI). Note the large fluorescein staining area superior to the disk (BI), two leaking spots at the inferotemporal quadrant and fluorescein accumulation in the subretinal space superior to the macula (B3). Post-treatment color fundus photography shows subsidence of bullous retinal detachment (A2). Decrease of fluorescein leakage (B2). Fluorescein staining remained with no obvious enlarging in FA (B4).

Abbreviation: FA, fluorescein angiography. initial choroidal vascular compromise leads to dysfunction in the overlying RPE, which eventually causes serous retinal detachment and exudation. ${ }^{11,12}$

Typical cases of CSC have been reported to spontaneously resolve in 1-3 months, ${ }^{13}$ without requiring particular treatment. In about $10 \%$ of cases, however, resolution of retinal detachment does not occur, leading to RPE damage. ${ }^{12,14,15}$ Since the RPE damage causes retinal and/or pigment epithelial detachments that determine the prognosis of visual recovery, ${ }^{16} \mathrm{CSC}$ without spontaneous resolution needs a proper and timely treatment. Typical CSC with a discrete leak point can be treated with photocoagulation, but chronic and atypical variants of CSC are sometimes difficult to treat. Moreover, to date, no standard treatment for atypical or excessive CSC has been established. Conventional photocoagulation cannot be used in subfoveal lesions. The grid laser photocoagulation for atypical CSC, reported by Yannuzzi et al, ${ }^{10}$ produced anatomical resolution in retinal detachments. However, it failed to improve the visual outcome.

Acute visual loss after photodynamic therapy was reported. Transient choroidal ischemia after photodynamic therapy (PDT) with verteporfin in AMD has been observed previously in human and animal studies. Subthreshold TTT, which could avoid damaging the overlying neural retina may not cause acute vision loss and could be applied to any patient. ${ }^{17}$ Furthermore, PDT is not practical in most countries because of economic reasons. TTT is a less expensive tool and has been recently reported to accelerate the resolution of CSC and improve the visual outcome. ${ }^{6,7}$ The present study also demonstrates that TTT may be a safer alternative treatment than PDT for atypical variants of CSC. The mechanism by which treatment with raised temperature leads to the repair of RPE damage has not been well understood. This report provides no information on this issue, either. However, it is certain that damage to photoreceptors has to be minimal to obtain a reasonable visual outcome. The laser beam with an $810 \mathrm{~nm}$ wavelength that is used in TTT is predominantly absorbed by pigmented cells and the choroid, whereas absorption by xanthophylls in photoreceptors is relatively low. ${ }^{18}$ This may explain the difference in the susceptibility to temperature increase between the sensory retina and choroid. TTT upregulates the expression of heat shock proteins, which protect the cells from various insults, ${ }^{19,20}$ including the choroidal layers. ${ }^{21}$ This also implies that although TTT may result in cellular damage in retinal layers,${ }^{22}$ the adverse effects can be minimized by carefully selecting the laser treatment variables of TTT for CSC. ${ }^{23}$ Standard photocoagulation that uses hyperthermia at $>45^{\circ} \mathrm{C}$ exerts a direct 
and irreversible cytotoxic effect resulting in cell necrosis, which causes scar formation and often neovascularization. Less invasive treatment by TTT may have an advantage over standard photocoagulation in atypical CSC when leaking points are multiple.

Our findings demonstrated the efficacy of TTT in atypical $\mathrm{CSC}$, and also suggests that it can be applied to typical CSC when serous retinal detachment superimposes the leaking point. This pilot study, however, demonstrates only short-term safety and efficacy. Because even clinically subthreshold TTT could damage the neural retina, ${ }^{24}$ the establishment of the appropriate parameters of TTT will be necessary. Long-term randomized controlled studies on a large scale are needed to determine the appropriate power and duration of TTT and ensure its safety.

\section{Disclosure}

The authors report no conflicts of interest in this work.

\section{References}

1. Journee-de Korver JG, Oosterhuis JA, Kakebeeke-Kemme HM, de WolffRouendaal D. Transpupillary thermotherapy (TTT) by infrared irradiation of choroidal melanoma. Doc Ophthalmol. 1992;82(3):185-191.

2. Othmane IS, Shields CL, Shields JA, Gunduz K, Mercado G. Circumscribed choroidal hemangioma managed by transpupillary thermotherapy. Arch Ophthalmol. 1999;117(1):136-137.

3. Rapizzi E, Grizzard WS, Capone A Jr. Transpupillary thermotherapy in the management of circumscribed choroidal hemangioma. Am J Ophthalmol. 1999;127(4):481-482.

4. Reichel E, Berrocal AM, Ip M, et al. Transpupillary thermotherapy of occult subfoveal choroidal neovascularization in patients with age-related macular degeneration. Ophthalmology. 1999;106(10):1908-1914.

5. Newsom RS, McAlister JC, Saeed M, McHugh JD. Transpupillary thermotherapy (TTT) for the treatment of choroidal neovascularisation. Br J Ophthalmol. 2001;85(2):173-178.

6. Hussain N, Khanna R, Hussain A, Das T. Transpupillary thermotherapy for chronic central serous chorioretinopathy. Graefes Arch Clin Exp Ophthalmol. 2006;244(8):1045-1051.

7. Shukla D, Kolluru C, Vignesh TP, Karthikprakash S, Kim R. Transpupillary thermotherapy for subfoveal leaks in central serous chorioretinopathy. Eye (Lond). 2008;22(1):100-106.

8. Tranos P, Singh M, Peter NM, Dhir L, Kon C, Rassam S. Transpupillary thermotherapy for the treatment of subfoveal choroidal neovascularization associated with age-related macular degeneration. Acta Ophthalmol Scand. 2004;82(5):585-590.

9. Gass JD. Bullous retinal detachment. An unusual manifestation of idiopathic central serous choroidopathy. Am J Ophthalmol. 1973;75(5): $810-821$.

Clinical Ophthalmology

\section{Publish your work in this journal}

Clinical Ophthalmology is an international, peer-reviewed journal covering all subspecialties within ophthalmology. Key topics include: Optometry; Visual science; Pharmacology and drug therapy in eye diseases; Basic Sciences; Primary and Secondary eye care; Patient Safety and Quality of Care Improvements. This journal is indexed on

Submit your manuscript here: http://www.dovepress.com/clinical-ophthalmology-journal
10. Yannuzzi LA, Slakter JS, Kaufman SR, Gupta K. Laser treatment of diffuse retinal pigment epitheliopathy. Eur J Ophthalmol. 1992;2(3):103-114.

11. Hayashi K, Hasegawa Y, Tokoro T. Indocyanine green angiography of central serous chorioretinopathy. Int Ophthalmol. 1986;9(1):37-41.

12. Iida T, Yannuzzi LA, Spaide RF, Borodoker N, Carvalho CA, Negrao S. Cystoid macular degeneration in chronic central serous chorioretinopathy. Retina. 2003;23(1):1-7.

13. Gilbert CM, Owens SL, Smith PD, Fine SL. Long-term follow-up of central serous chorioretinopathy. Br J Ophthalmol. 1984;68(11): $815-820$.

14. Brancato R, Scialdone A, Pece A, Coscas G, Binaghi M. Eight-year follow-up of central serous chorioretinopathy with and without laser treatment. Graefes Arch Clin Exp Ophthalmol. 1987;225(3):166-168.

15. Levine R, Brucker AJ, Robinson F. Long-term follow-up of idiopathic central serous chorioretinopathy by fluorescein angiography. Ophthalmology. 1989;96(6):854-859.

16. Loo RH, Scott IU, Flynn HW Jr, et al. Factors associated with reduced visual acuity during long-term follow-up of patients with idiopathic central serous chorioretinopathy. Retina. 2002;22(1):19-24.

17. Costa RA, Farah ME, Cardillo JA, Calucci D, Williams GA. Immediate indocyanine green angiography and optical coherence tomography evaluation after photodynamic therapy for subfoveal choroidal neovascularization. Retina. 2003;23(2):159-165.

18. Gunduz K. Transpupillary thermotherapy in the management of circumscribed choroidal hemangioma. Surv Ophthalmol. 2004; 49(3):316-327.

19. Polla BS, Stubbe H, Kantengwa S, Maridonneau-Parini I, JacquierSarlin MR. Differential induction of stress proteins and functional effects of heat shock in human phagocytes. Inflammation. 1995;19(3):363-378.

20. Kim HJ, Hwang NR, Lee KJ. Heat shock responses for understanding diseases of protein denaturation. Mol Cells. 2007;23(2):123-131.

21. Desmettre T, Maurage CA, Mordon S. Heat shock protein hyperexpression on chorioretinal layers after transpupillary thermotherapy. Invest Ophthalmol Vis Sci. 2001;42(12):2976-2980.

22. She H, Li X, Yu W. Subthreshold transpupillary thermotherapy of the retina and experimental choroidal neovascularization in a rat model. Graefes Arch Clin Exp Ophthalmol. 2006;244(9):1143-1151.

23. Ming Y, Algvere PV, Odergren A, et al. Subthreshold transpupillary thermotherapy reduces experimental choroidal neovascularization in the mouse without collateral damage to the neural retina. Invest Ophthalmol Vis Sci. 2004;45(6):1969-1974.

24. Morimura Y, Okada AA, Hayashi A, et al. Histological effect and protein expression in subthreshold transpupillary thermotherapy in rabbit eyes. Arch Ophthalmol. 2004;122(10):1510-1515.

\section{Dovepress}

PubMed Central and CAS, and is the official journal of The Society of Clinical Ophthalmology (SCO). The manuscript management system is completely online and includes a very quick and fair peer-review system, which is all easy to use. Visit http://www.dovepress.com/ testimonials.php to read real quotes from published authors. 